\title{
Combined target site $(k d r)$ mutations play a primary role in highly pyrethroid resistant phenotypes of Aedes aegypti from Saudi Arabia
}

Ashwaq M. Al Nazawi ${ }^{1,2^{*}}$, Jabir Aqili², Mohammed Alzahrani' ${ }^{2}$ Philip J. McCall ${ }^{1}$ and David Weetman ${ }^{1}$

\begin{abstract}
Background: Pyrethroid resistance is a threat to effective vector control of Aedes aegypti, the vector of dengue, Zika and other arboviruses, but there are many major knowledge gaps on the mechanisms of resistance. In Jeddah and Makkah, the principal dengue-endemic areas of Saudi Arabia, pyrethroids are used widely for Ae. aegypti control but information about resistance remains sparse, and the underlying genetic basis is unknown. Findings from an ongoing study in this internationally significant area are reported here.

Methods: Aedes aegypti collected from each city were raised to adults and assayed for resistance to permethrin, deltamethrin (with and without the synergist piperonyl butoxide, PBO), fenitrothion, and bendiocarb. Two fragments of the voltage-gated sodium channel (Vgsc), encompassing four previously identified mutation sites, were sequenced and subsequently genotyped to determine associations with resistance. Expression of five candidate genes (CYP9J10, CYP9J28, CYP9J32, CYP9M6, ABCB4) previously associated with pyrethroid resistance was compared between assay survivors and controls.

Results: Jeddah and Makkah populations exhibited resistance to multiple insecticides and a similarly high prevalence of resistance to deltamethrin compared to a resistant Cayman strain, with a significant influence of age and exposure duration on survival. PBO pre-exposure increased pyrethroid mortality significantly in the Jeddah, but not the Makkah strain. Three potentially interacting Vgsc mutations were detected: V1016G and S989P were in perfect linkage disequilibrium in each strain and strongly predicted survival, especially in the Makkah strain, but were in negative linkage disequilibrium with 1534C, though some females with the Vgsc triple mutation were detected. The candidate gene CYP9J28 was significantly over-expressed in Jeddah compared to two susceptible reference strains, but none of the candidate genes was consistently up-regulated to a significant level in the Makkah strain.

Conclusions: Despite their proximity, Makkah and Jeddah exhibit significant differences in pyrethroid resistance phenotypes, with some evidence to suggest a different balance of mechanisms, for example with more impact associated with CYP450s in the Jeddah strain, and the dual kdr mutations 989P and 1016G in the more resistant Makkah strain. The results overall demonstrate a major role for paired target site mutations in pyrethroid resistance and highlight their utility for diagnostic monitoring.
\end{abstract}

Keywords: Dengue, Saudi Arabia, Aedes aegypti, Insecticide resistance, Piperonyl butoxide (PBO), Knockdown resistance

\footnotetext{
*Correspondence: A.Alnazawi@liverpool.ac.uk

'Department of Vector Biology, Liverpool School of Tropical Medicine,

Pembroke Place, Liverpool L3 5QA, UK

${ }^{2}$ Saudi Ministry of Health, Riyadh, Saudi Arabia
}

(c) The Author(s). 2017 Open Access This article is distributed under the terms of the Creative Commons Attribution 4.0 International License (http://creativecommons.org/licenses/by/4.0/), which permits unrestricted use, distribution, and reproduction in any medium, provided you give appropriate credit to the original author(s) and the source, provide a link to the Creative Commons license, and indicate if changes were made. The Creative Commons Public Domain Dedication waiver (http://creativecommons.org/publicdomain/zero/1.0/) applies to the data made available in this article, unless otherwise stated. 


\section{Background}

Dengue virus is transmitted by the bite of Aedes mosquitoes, especially Ae. aegypti, has recently increased dramatically in prevalence and now affects more than 100 tropical and sub-tropical countries, with half of the world's population at risk $[1,2]$. Although a licensed vaccine against the four dengue serotypes recently became available [3], and already is approved in some endemic countries [4, 5], protection is incomplete and unequal across serotypes [6]. Hence, Aedes control using insecticides will remain a key intervention for dengue prevention, especially given the added benefit of simultaneously targeting other Aedestransmitted arboviruses including chikungunya and Zika. In Saudi Arabia, several areas are endemic regions for the dengue virus of which Jeddah and Makkah are by far the most important (Fig. 1). First reported in Jeddah in 1994, three serotypes DENG-1, DENG-2, DENG-3 [7] have been confirmed, and 9,096 cases were diagnosed between 2013 and 2015, with a further 3,035 in Makkah. Multiple insecticides are applied to target immature vector stages in Makkah and Jeddah, but pyrethroids are the most commonly used to control adult $A e$. aegypti by indoor and outdoor space spraying. The efficacy of pyrethroidbased adult control has been found to be impacted by insecticide resistance in Ae. aegypti from diverse locations [8, 9]. Information on the insecticide resistance status of Saudi Arabian populations of Ae aegypti remains limited $[10,11]$, and is absent for Jeddah, the primary national centre for dengue.
The most common mechanisms of insecticide resistance in Ae. aegypti involve target site alterations and metabolic resistance, comprising altered activity of enzymes from three superfamilies, esterases (CCEs), glutathione-s-transferases (GSTs) and cytochrome P450s (CYP450s), although several other enzyme families are likely to be involved [12]. Overexpression of Ae. aegypti CYP450s, especially from the CYP9 and CYP6 subfamilies has frequently been implicated in pyrethroid resistant phenotypes [13], and several overexpressed P450s have been shown to metabolise pyrethroids in vitro [14-16]. Pyrethroid and DDT target site resistance results from amino acid substitutions in the voltage-gated sodium channel (Vgsc) that usually reduce insecticide binding, although other mechanisms unrelated to binding are known $[17,18]$. Some mutations in the insect Vgsc can prevent the normal action of pyrethroids and DDT (repetitive nerve firing, paralysis and death) leading to knockdown resistance $(k d r)$ [18]. Multiple $k d r$ mutations have been recorded in Ae. aegypti worldwide including G923V, L982W, I1011M/V, S989P, V1016G/I, F1534C and D1763Y [19, 20]. Of these, I1011M, V1016G/I [21], S989P [22] and F1534C [23, 24] either individually or in combination have been associated directly with pyrethroid resistance. The vast majority of studies of resistance mechanisms in Ae. aegypti are from The Americas and Asia [19, 25, 26], though a recent first study from West Africa identified the presence of target site resistance shared with American populations [27]. Knowledge of resistance mechanisms in the Middle East and their relationship with other regions is currently

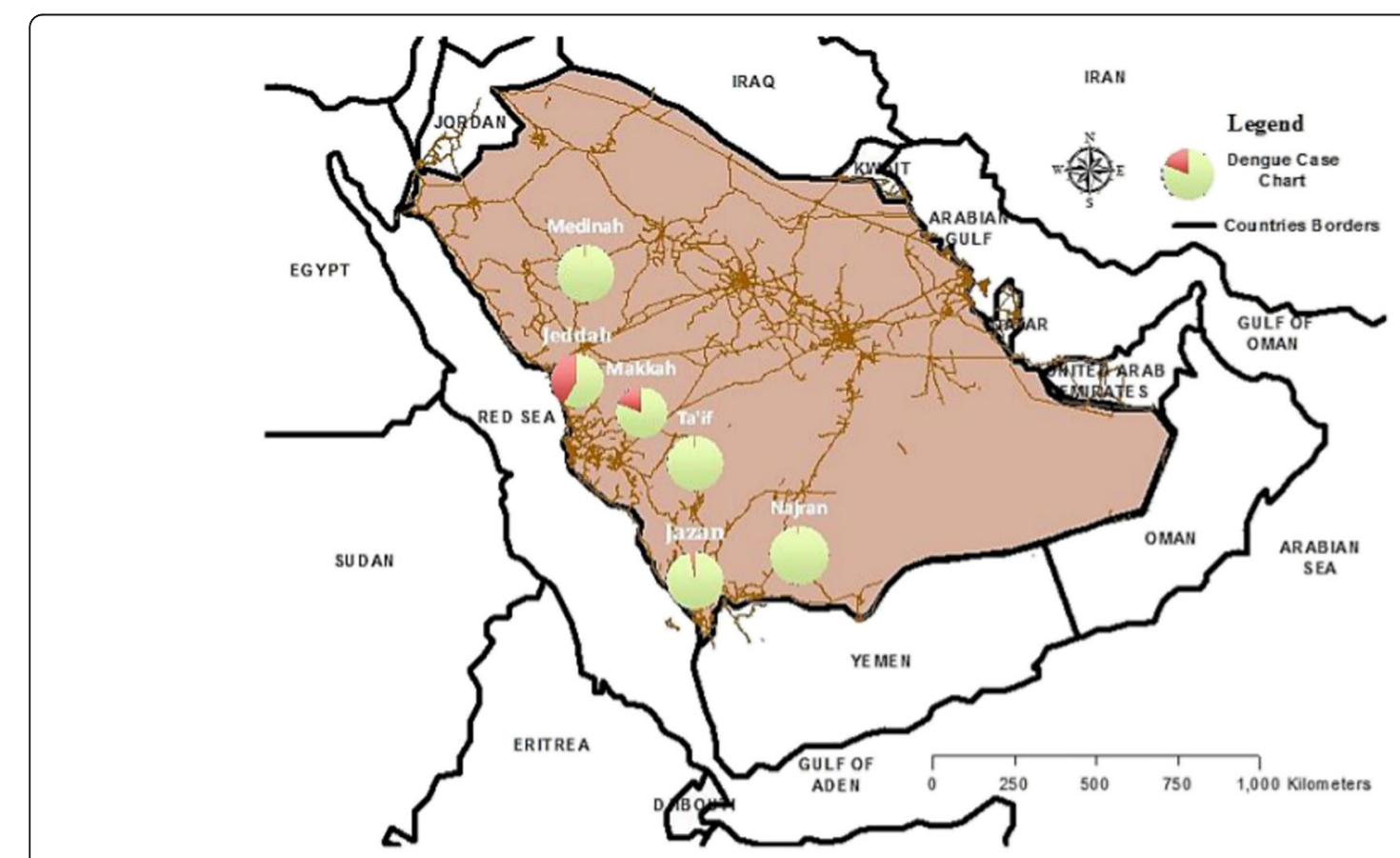

Fig. 1 Dengue fever cases in cities of Saudi Arabia from 2013-2015 
lacking. This paper investigates the resistance status of Ae. aegypti from Jeddah and Makkah, and focuses in particular on pyrethroid resistance and its underlying mechanisms as compared with established resistant and susceptible strains.

\section{Methods}

\section{Mosquito strains, collection and rearing}

The first collection in Jeddah $\left(21^{\circ} 63^{\prime} 1.244^{\prime \prime} \mathrm{N}, 39^{\circ} 19^{\prime}\right.$ $\left.8.71^{\prime \prime W}\right)$ and Makkah $\left(21^{\circ} 45^{\prime} 8.36^{\prime \prime} \mathrm{N}, 39^{\circ} 78^{\prime} 6.98^{\prime \prime} \mathrm{E}\right)$ was made in December to April (Makkah) and June to August 2015 (Jeddah). In both areas, 50 ovitraps were set targeting sites in which dengue cases have been reported. In Makkah, collections were made from private farms, brick factories, car and tyre shops, fuel stations and residential houses. Similarly, in Jeddah traps were set in buildings under construction, in shops and houses. All traps were checked every two days and collected after five days. The eggs were dried, preserved at room temperature in a sealed plastic bag and shipped to the Liverpool School of Tropical Medicine (LSTM). Egg batches were hatched, and larvae reared to adults on a diet of chinchilla pellets under insectary conditions of $27{ }^{\circ} \mathrm{C}$ and $70 \%$ relative humidity with a constant photoperiod (12 light:12 h dark). Adult females, 3-5 days old, from the first laboratory generation (i.e. the offspring of the adults from the egg collections, hereafter referred to as ' $\mathrm{F} 1$ females'), which had been fed ad libitum on 10\% sugar solution were used for phenotypic bioassays and characterization of molecular resistance mechanisms. The Cayman strain [23] used as a reference resistant strain and the standard strains New Orleans and Rockefeller as susceptible references (used for qPCR only); all strains were raised under the same conditions. A second, broader field collection in March-April 2016 targeted larvae present in water coolers, barrels, buckets and water containers such as under air conditioners and buildings under construction from a wide area within Jeddah and Makkah (Additional file 1: Figure S1). The larvae were reared at the local Municipal Insectaries on a diet of yeast under insectary conditions of $27{ }^{\circ} \mathrm{C}$ and $75 \%$ relative humidity with a constant photoperiod (12 light: $12 \mathrm{~h}$ dark) and females (hereafter referred to as 'field females'), fed ad libitum on $10 \%$ sugar solution, were bioassayed at $3-5$ days.

\section{Phenotypic assays}

WHO tube bioassays were performed with a minimum of four replicates plus control, with approximately 25 females per tube. Using the standard $\mathrm{WHO}$ protocol [28], female mosquitoes were exposed to permethrin (0.75\%), deltamethrin $(0.05 \%)$, fenitrothion $(1.0 \%)$ or bendiocarb $(0.1 \%)$ for $60 \mathrm{~min}$, under the insectary conditions described above, and then transferred to recovery tubes with access to $10 \%$ sugar solution. The mortality rate was recorded following $24 \mathrm{~h}$ exposure. Owing to logistical limitations, bendiocarb was not tested in the field females. F1 female mosquitoes were also exposed to deltamethrin after exposure to the synergist $4 \%$ piperonyl butoxide (PBO), which amongst other potential effects, is intended to block the action of CYP450s. We used the following procedure: $1 \mathrm{~h}$ control paper $+1 \mathrm{~h}$ deltamethrin, $1 \mathrm{~h} \mathrm{PBO}+1 \mathrm{~h}$ deltamethrin, $1 \mathrm{~h} \mathrm{PBO}+1 \mathrm{~h}$ control; $2 \mathrm{~h}$ control paper, with mortality recorded after $24 \mathrm{~h}$ as before. The level of resistance to deltamethrin was investigated using bioassays with longer durations of exposure, in which the impact of age on mortality was also examined. Three-to-five and tenday-old F1 adult females from the Jeddah, Makkah and Cayman strains were exposed for $1 \mathrm{~h}, 6 \mathrm{~h}$ or $8 \mathrm{~h}$, with mortality recorded $24 \mathrm{~h}$ after the bioassay.

\section{Target site mutations}

Genomic DNA was extracted from F0 females from the Jeddah $(N=26)$, and Makkah $(N=15)$ strains using Nexttec kits $\left(\right.$ Nexttec $^{\mathrm{Tw}}$, Biotechnologie $\mathrm{GmbH}$, Hilgertshausen, Germany) according to the manufacturer's instructions. PCR primers (IIS5-6 (3) F: 5'-ATC GCT TCC CGG ACA AAG AC-3'; IIS5-6 (3) R: 5'-GTT GGC GAT GTT CGA CTT GA-3') were designed using Primer 3 $[29,30]$ to amplify $k d r$ mutations in the sixth transmembrane segment of domain II of the Vgsc, which includes the resistance-associated codons 989, 1,011, 1,016. For the Makkah samples a segment of domain III that includes codon 1,534 we amplified exon 30 using the primers AaNa31F and AaNa31R [23]. PCR reactions were carried out in a $25 \mu \mathrm{l}$ reaction volume containing $12.5 \mu \mathrm{l}$ TaqRed Mix (Bioline, London, UK), $0.5 \mu \mathrm{l}$ forward and reverse primers $(10 \mu \mathrm{M})$ and $2 \mu \mathrm{l}$ of DNA extract. Cycling conditions for the domain II primers were $95{ }^{\circ} \mathrm{C}$ for $5 \mathrm{~min}$, 35 cycles of $\left(94{ }^{\circ} \mathrm{C}\right.$ for $30 \mathrm{~s}, 65{ }^{\circ} \mathrm{C}$ for $30 \mathrm{~s}, 72{ }^{\circ} \mathrm{C}$ for $1 \mathrm{~min}$ ) and a $10 \mathrm{~min}$ final elongation step at $72{ }^{\circ} \mathrm{C}$. For the domain III primers, conditions were the same except that the annealing temperature was $62{ }^{\circ} \mathrm{C}$ for $30 \mathrm{~s}$. PCR products were purified using the QIAquick ${ }^{\circ}$ PCR Purification Kit (Qiagen, Manchester, UK) and sequenced commercially (Source Bioscience, Rochdale, UK). Sequence data were assembled and aligned using Codon Code Aligner version 4.2.7. A TaqMan SNP genotyping assay for F1534C was designed and compared to an existing PCR tetraplex assay [23] and validated by comparison with DNA sequencing of field-caught Jeddah and Makkah mosquitoes. TaqMan reactions were performed in a $10 \mu \mathrm{l}$ final volume containing $5 \mu \mathrm{l}$ of $\mathrm{TaqMan}^{\circ}$ Gene Expression SensiMix (Applied Biosystem, Foster city, USA), $0.125 \mu \mathrm{l}$ primer/probe, $3.875 \mu \mathrm{l}$ sterile water (Sigma, Gillingham, Dorset, UK) and $1 \mu \mathrm{l}$ of DNA. Assays were run on an Agilent MX3000P qPCR thermal cycler under the following conditions: a $10 \mathrm{~min}$ cycle at $95{ }^{\circ} \mathrm{C}$, and 40 cycles of $92{ }^{\circ} \mathrm{C}$ for $15 \mathrm{~min}$ and $60{ }^{\circ} \mathrm{C}$ for 
$1 \mathrm{~min}$. Based on 21 samples, the concordance between the TaqMan SNP screening results and DNA sequencing analysis was $100 \%$. Results were concordant between assays in 19 of 21 samples with remaining two genotypes giving an unclear score using the tetraplex method. To investigate the association between target site mutations and survival, F2 females from the Jeddah and Makkah strains were exposed to deltamethrin in WHO bioassays for either a standard $1 \mathrm{~h}$ duration and for longer periods of 4-6 h. Genotypes of females killed by $1 \mathrm{~h}$ of deltamethrin exposure (susceptible), were compared with survivors of the longer exposures.

\section{Gene expression}

Expression profiles of genes previously associated with Ae. aegypti pyrethroid resistance, four CYP450 genes (CYP9J10, CYP9J28, CYP9J32, CYP9M6) and an ABC transporter $(A B C B 4)[16,31,32]$ (Additional file 2: Table S1) were assessed using quantitative reverse transcription PCR (qRT-PCR), in relation to two susceptible strains, New Orleans and Rockefeller. Total RNA was extracted from three pools of 5 female mosquitoes in each strain, which had not been exposed to insecticide, using the Ambion RNAqueous ${ }^{\oplus}$ Kit (Life Technologies, Paisley, UK), with the quantity of RNA yields assessed using a Nanodrop ND-1000 (Thermo Scientific, Delaware, USA). Synthesis of cDNA used Superscript III (Invitrogen, Carlsbad, CA, USA) according to the manufacturer's guidelines, and cDNA was purified using a QIAquick spin column (QIAuick PCR Purification Kit, Qiagen). The qRTPCR reactions were performed in a volume of $10 \mu \mathrm{l}$ with $5 \mu \mathrm{l}$ (Applied Biosystems, Texas, USA), $0.4 \mu \mathrm{l}$ forward and reverse primer $(10 \mu \mathrm{M}), 3.2 \mu \mathrm{l} \mathrm{ddH}_{2} \mathrm{O}$, and $1 \mu \mathrm{l}$ of cDNA (approximately $2 \mathrm{ng}$ ), under the following conditions: $95{ }^{\circ} \mathrm{C}$ for $3 \mathrm{~min}$, followed by 40 cycles of $95{ }^{\circ} \mathrm{C}$ for $10 \mathrm{~s}$ and $60{ }^{\circ} \mathrm{C}$ for $10 \mathrm{~s}$. The relative expression level and fold change (FC) of each candidate gene relative to the susceptible strains was calculated using the $\Delta \Delta \mathrm{cT}$ method [33] after normalisation with two housekeeping genes, RPS3 (ribosomal protein S3) and the 60 S ribosomal protein L8. All primer sequences and their origins are shown in Additional file 2: Table S1.

\section{Statistical analyses}

Effects of strains, age, and exposure duration or of strain and synergist exposure were analysed using generalised linear models with binomial link functions in SPSS v22. Pearson chi-square tests were used to assess associations between $k d r$ mutations and phenotypes in genotypic and allelic tests, with odds ratios used to measure effect size. Error bars represent 95\% confidence intervals calculated using the method of Wilson, with continuity correction [34]. Haploview [35] was used to estimate haplotype frequencies and perform haplotype association tests for the $k d r$ mutations. Two-tailed t-tests were used to compare candidate gene expression levels between strains: significance was accepted only when detected between a resistant strain and both susceptible strains.

\section{Results}

\section{Phenotypic resistance}

Bioassays on field and laboratory strains of female $A e$. aegypti from Jeddah and Makkah indicated a high prevalence of resistance to permethrin and deltamethrin, and also to bendiocarb (tested in F1 females only). Qualitatively, results from the lab strains and field samples were comparable, despite their differing also in collection date and precise sample location. In some cases, mortalities were significantly higher in the field than F1 females (Fig. 2). A high prevalence of resistance to each pyrethroid was found in both Jeddah and Makkah and was especially pronounced for permethrin assays in which fewer than $10 \%$ of exposed females died. Similarly, mortality from bendiocarb exposure (tested in F1 females only) was negligible in each strain. For fenitrothion, assays on field samples revealed suspected evidence of resistance (mortality 90-97\%) in Makkah, or confirmed resistance in the Makkah F1 strain, whereas in Jeddah field and F1, the results were more consistent and suggested borderline resistance $(\mathrm{F} 1=92 \%$; field $=98 \%)$. Although very similar for permethrin and bendiocarb, and equivocal for fenitrothion (owing to F1 vs field variation in Makkah), the prevalence of deltamethrin resistance was higher in Makkah than Jeddah females, whether tested on either F1 $\left(\chi^{2}=12.26, d f=1, P=0.0005\right)$ or field females $\left(\chi^{2}=35.94\right.$, $d f=1, P<0.0001)$. PBO bioassays significantly improved susceptibility to deltamethrin (Fig. 3; Additional file 2: Table S2) and whilst the GLiM strain $\times$ PBO interaction term was not significant, only in the Jeddah strain $\left(\chi^{2}=\right.$ $12.17, d f=1, P=0.0005)$, was the mortality significantly elevated (Makkah F1 strain, $X^{2}=2.26, d f=1, P=0.13$; Cayman strain; $\chi^{2}=3.41, d f=1, P=0.065$ ).

The level of mortality and its dependence on female age was assessed in each of the three strains by exposing either 3-5 day-old or 10 days-old to deltamethrin for progressively longer periods of $(1 \mathrm{~h}, 6 \mathrm{~h}, 8 \mathrm{~h})$. Strain, age, and exposure were all significant in the GLiM, but effects on mortality were not straightforward, as evident from the significance of all three two-way interaction terms (Fig. 4; Additional file 2: Table S3). In both Saudi strains, the mortality was low following short exposure, though somewhat higher in older females, but mortality was much higher after longer exposures, irrespective of age (70$100 \%)$. In contrast, young females of the reference resistant strain Cayman exhibited no difference in mortality across exposure durations but when older females were tested longer exposures induced greater mortality (Fig. 4). 

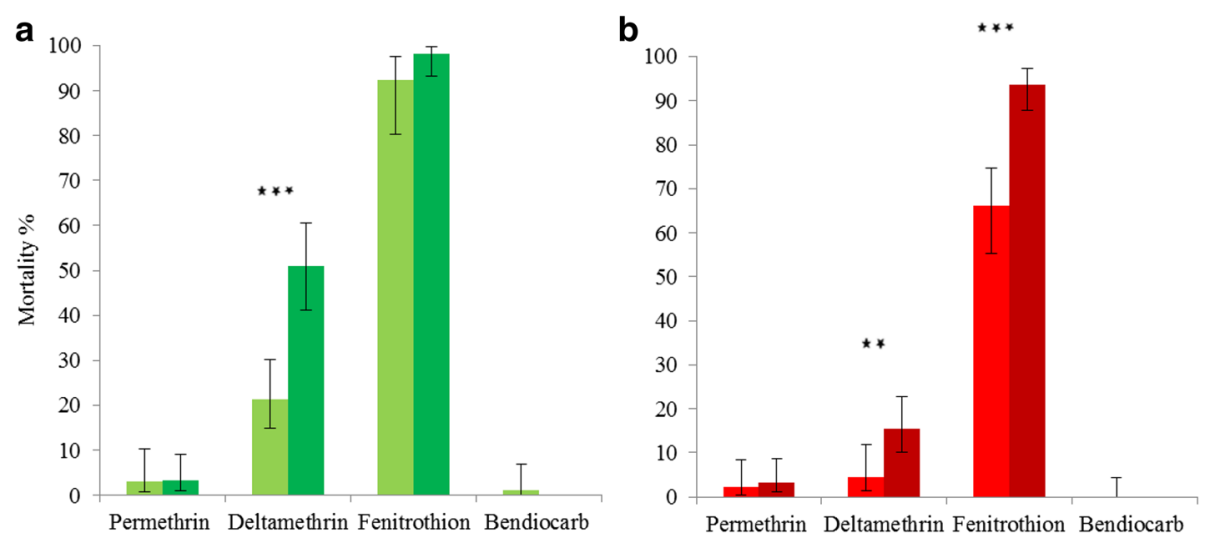

Fig. 2 Susceptibility status of female Ae. aegypti to insecticides in 60 min bioassays with exposure to permethrin, deltamethrin, fenitrothion and bendiocarb. a Jeddah laboratory strain (light green) and field strain (dark green). b Makkah laboratory strain (light red) and field strain (dark red). Statistical significance is indicated by ${ }^{*} P<0.05,{ }^{*} P<0.01$ and ${ }^{* *} P<0.001$. Error bars are $95 \%$ confidence intervals

\section{Molecular mechanisms}

DNA sequencing from wild (F0) mosquitoes of Jeddah and Makkah populations revealed three $k d r$ substitutions in the Vgsc: S989P, V1016G and F1534C but neither of the I1011M or I1011V mutations (GenBank Accession Nos. S989P_V1016G: KY626180-KY626197, GenBank Accession Nos F1534C: KY046222-KY046237). The V1016G and S989P mutations were in perfect linkage disequilibrium (LD) in the Jeddah $(n=26)$ and Makkah ( $n=15)$ F0 collections, with allele frequency estimates of 0.46 (95\% CI: 0.33-0.59) and 0.67 (95\% CI: 0.49-0.81), respectively. The allele frequency of $1534 \mathrm{C}$ was 0.5 (95\% CI: 0.37- 0.63) in Jeddah and 0.4 (95\% CI: 0.250.58) in Makkah and F1534C was in strong, but imperfect, repulsive LD with S989P/V1016G $\left(r^{2}=0.59\right.$ in Jeddah and 0.87 in Makkah), with five Jeddah females and one Makkah female possessing one chromosome each with the triple

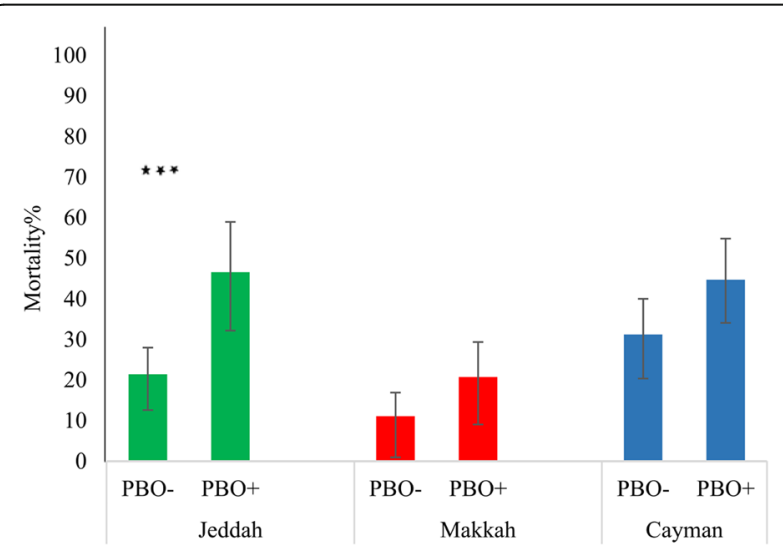

Fig. 3 Deltamethrin 60 min bioassays with and without $60 \mathrm{~min}$ pre-exposure to the synergist $\mathrm{PBO}(\mathrm{PBO}+, \mathrm{PBO}$ - respectively). Statistical significance is indicated by ${ }^{*} P<0.05$, ${ }^{* *} P<0.01$ and ${ }^{* *} P<0.001$. Error bars are $95 \%$ confidence intervals mutant $(989 \mathrm{P}+1016 \mathrm{G}+1534 \mathrm{C})$ haplotype (Additional file 3: Table S4).

To evaluate impacts of the mutations on deltamethrin survival, haplotypes (estimated from genotypic data) of mosquitoes killed by duration WHO susceptibility bioassays ( $<20 \%$ mortality in each strain) were compared with survivors of long 4-6 h bioassays ( $\geq 60 \%$ mortality in each strain). Four haplotypes were estimated in the two populations (Table 1) of which: 989P + 1016G + F1534 was strongly associated with resistance and S989+V1016+1534C with susceptibility. The triple mutant 989P + 1016G + $1534 \mathrm{C}$ was somewhat more common in susceptible females, but the estimated frequencies were very low. Six distinct and one ambiguous triple-locus genotype could be discerned though not all were present in both Makkah and Jeddah. In concordance with the haplotypic tests, the double mutant genotype 989P/P + 1016G/G + $1534 \mathrm{~F} / \mathrm{F}$ and single mutant genotype $989 \mathrm{~S} / \mathrm{S}+1016 \mathrm{~V} / \mathrm{V}+$ $1534 \mathrm{C} / \mathrm{C}$ (numbers 1 and 2 in Table 2) differed strongly in the effect on resistance $\left(\chi^{2}=27.7, d f=1, P<0.0001\right.$; OR $=$ 79.2, 95\% CI: 12-522). Though relatively rare, none of the six individual possessing genotypes which must have contained a triple mutant allele (i.e. 989P + 1016G + 1534C; genotype numbers 6 and 7) survived exposure, suggesting a lack of resistance conferred by this allele when heterozygous. Interestingly the triple heterozygote genotype (number 5) showed significant association with resistance $\left(\chi^{2}=17.4, d f=1, P<0.0001\right.$; OR $=29.2,95 \% \mathrm{CI}$ : 5-164), which was approximately 2.7 times lower than the most resistant genotype (number 1 ), though not significantly so $(P=0.36)$.

Quantitative real-time PCR in all strains (each unexposed to insecticide) showed several genes to be more highly expressed than in the New Orleans colony. However, when considering comparisons with Rockefeller, fewer of the candidate genes were significantly upregulated (Fig. 5). In the 

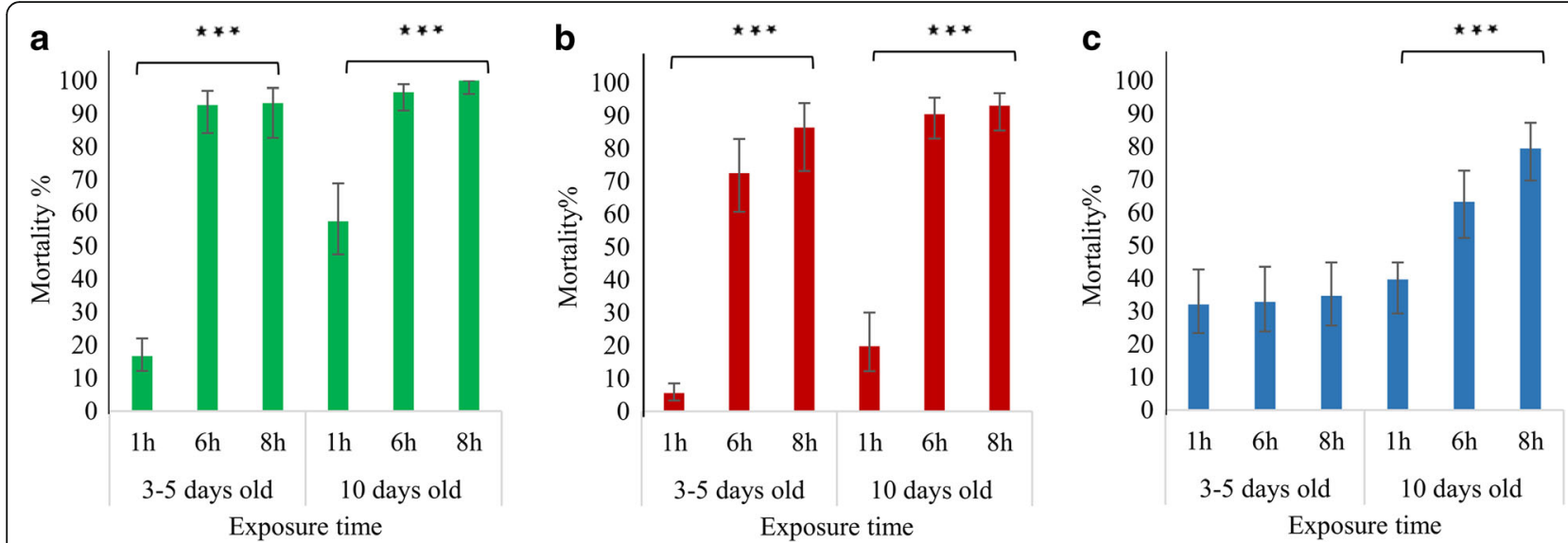

Fig. 4 Impacts of age and exposure duration on deltamethrin survivorship in a Jeddah (green), b Makkah (red) and c Cayman (blue). Statistically significant variation among exposure times (ANOVA) is indicated by ${ }^{*} P<0.05,{ }^{* *} P<0.01$ and ${ }^{* * *} P<0.001$. Error bars represent the $95 \%$ confidence intervals

Makkah strain only $A B C B 4$ neared significance, and in the Jeddah strain, CYP9J28 and CYP9J10 were significantly and consistently overexpressed to high levels, and in the Cayman strain, the CYP9M6 gene was over-expressed compared to both susceptible colonies. In each case, expression levels of the four P450 genes were lower in Makkah than in Jeddah.

\section{Discussion}

Physiological resistance to pyrethroids in adult Ae. aegypti has now been detected around the globe with reports from Brazil, Mexico, Thailand, China, Grand Cayman, Latin America, Indonesia and Malaysia [26]. Reports of resistance from the Middle-Eastern region involve studies from the Ibb region of Yemen [36], from Jazan in Saudi Arabia [11] approximately $500 \mathrm{~km}$ north, and from Makkah [10], a further $700 \mathrm{~km}$ north. WHO bioassay results in each case were similar, with mortalities ranging from $75-93 \%$ to different pyrethroids. The previous study from Makkah [10] performed collections in 2008 and our data, which was gathered using the same methodology, suggests that in the intervening years, resistance to both permethrin and deltamethrin $(<20 \%$ mortality in our results) may have increased substantially though we note that in the absence of repeated samples, consistency of the bioassay data, and thus stability of this longer-term variation, cannot be assessed. Interestingly, despite the proximity of the sites, - Jeddah is less than $100 \mathrm{~km}$ from Makkah - standard 60 min assay deltamethrin resistance was significantly lower in Jeddah, although was still higher than in the Cayman reference resistant strain. These observations demonstrate a worrying temporal increase in resistance in Makkah together with unpredictability in the spatial scale over which resistance may vary, suggesting the involvement of local pressures rather than wider features of Ae. aegypti population genetic structure. In common with previous reports from Jazan province [11], Makkah and Jeddah strains showed high resistance to carbamate (benidocarb), but mortality following exposure to the organophosphate fenitrothion was only slightly reduced.

Age may also be an important factor in resistance and is crucial because older mosquitoes are more likely to transmit disease. In common with previous studies on Ae. aegypti and other mosquitoes [37, 38] ten-day-old females from Makkah and Jeddah were significantly more susceptible to deltamethrin than 3-5 days olds, though with a standard $60 \mathrm{~min}$ exposure the majority still survived. With long exposure times of six or eight hours up to $30 \%$ survival was observed, demonstrating that not only are most of the population classed as resistant (from $60 \mathrm{~min}$ bioassay data), but a small proportion is highly resistant, even when tested as older females. Surprisingly, given the slightly lower prevalence of resistance at

Table 1 Estimated haplotype frequencies and their association with deltamethrin resistance in Ae. aegypti from each strain

\begin{tabular}{|c|c|c|c|c|c|c|}
\hline \multirow[b]{2}{*}{ Haplotype } & \multicolumn{3}{|l|}{ Jeddah } & \multicolumn{3}{|l|}{ Makkah } \\
\hline & Susceptible & Resistant & $P$-value & Susceptible & Resistant & $P$-value \\
\hline 989P/1016G/F1534 & 0.187 & 0.628 & $6 \times 10^{-6}$ & 0.143 & 0.853 & $2 \times 10^{-8}$ \\
\hline S989/N1016/1534C & 0.655 & 0.338 & 0.002 & 0.786 & 0.147 & $4 \times 10^{-7}$ \\
\hline S989/V1016/F1534 & 0.079 & 0.030 & 0.319 & 0.036 & 0.000 & 0.269 \\
\hline 989P/1016G/1534C & 0.079 & 0.004 & 0.096 & 0.036 & 0.000 & 0.269 \\
\hline
\end{tabular}


Table 2 Triple-locus kdr genotypes, shown as amino acids at codons 989, 1,016 and 1,534 and their frequencies in mosquitoes surviving a long (4-6 h) deltamethrin exposure or killed by a $1 \mathrm{~h}$ exposure

\begin{tabular}{|c|c|c|c|c|c|c|c|c|c|c|}
\hline \multirow{2}{*}{\multicolumn{2}{|c|}{ Genotype }} & \multicolumn{2}{|c|}{ Jeddah } & \multicolumn{2}{|c|}{ Makkah } & \multicolumn{2}{|c|}{ Combined } & \multirow[b]{2}{*}{ Mortality } & \multirow[b]{2}{*}{$\mathrm{LCL}$} & \multirow[b]{2}{*}{$\mathrm{UCL}$} \\
\hline & & Alive & Dead & Alive & Dead & Alive & Dead & & & \\
\hline 1 & PGF/PGF & 7 & 3 & 12 & 0 & 19 & 3 & 0.14 & 0.04 & 0.36 \\
\hline 2 & SVC/SVC & 2 & 16 & 0 & 9 & 2 & 25 & 0.93 & 0.74 & 0.99 \\
\hline 3 & SVF/SVC & 0 & 4 & 0 & 0 & 0 & 4 & 1.00 & 0.40 & 1.00 \\
\hline 4 & SVF/PGF & 1 & 1 & 0 & 1 & 1 & 2 & 0.67 & 0.13 & 0.98 \\
\hline 5 & SVF, SVC/PGF, PGC & 9 & 3 & 5 & 3 & 14 & 6 & 0.30 & 0.13 & 0.54 \\
\hline 6 & SVC/PGC & 0 & 3 & 0 & 1 & 0 & 4 & 1.00 & 0.40 & 1.00 \\
\hline 7 & PGF/PGC & 0 & 2 & 0 & 0 & 0 & 2 & 1.00 & 0.20 & 1.00 \\
\hline
\end{tabular}

Mutant amino acids are shown in bold type. LCL and UCL are 95\% binomial confidence limits. Note that for genotype 5 alleles could not be determined unambiguously and alternates are shown

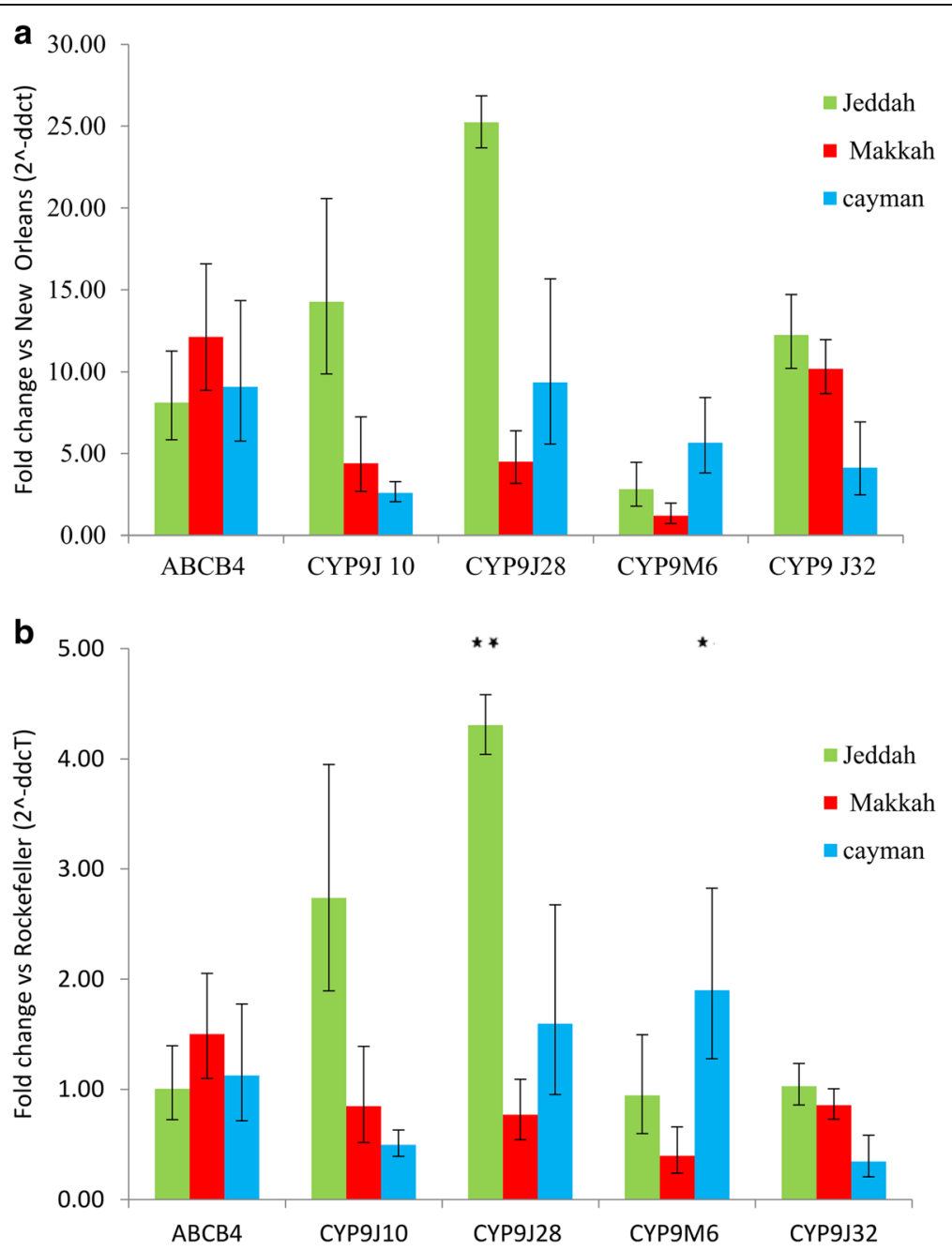

Fig. 5 Quantitative PCR analysis of candidate genes. Relative-fold changes compared to two susceptible strains a New Orleans, b Rockefeller are shown following normalisation to two endogenous reference genes. Error bars represent $95 \%$ confidence intervals. Significance is indicated for Rockefeller only where New Orleans is also significant $\left.{ }^{*} P<0.05,{ }^{* *} P<0.01\right)$ 
60 min, Cayman females of either age class survived long exposures better, suggesting a dissociation between prevalence and level of resistance, as documented in Anopheles gambiae [39].

Females from Jeddah suffered a significant increase in deltamethrin mortality, which more than doubled following pre-exposure to PBO. For Makkah (and Cayman) the increase was slight and not significant in either case, although the difference among populations was not large enough for detection of statistically significant heterogeneity. This contrasts with the almost complete synergy of deltamethrin by PBO (from approximately 5 to $98 \%$ mortality) reported by Bingham et al. in the Nha Trang Ae. aegypti strain from Vietnam [40], which, together with overexpression of the strongly pyrethroid-metabolising gene CYP9J32 [14] relative to the susceptible Bora Bora strain, was interpreted as evidence for a dominant role of CYP450 enzymes in deltamethrin resistance. While the impact of $\mathrm{PBO}$ was clear, some caution is required in causal links with CYP450s because, while the action of P450s can be blocked, as demonstrated directly in vivo in Ae. aegypti [16] other effects such as reduced cuticular penetration may occur [41]. Moreover, comparison with a single susceptible strain may be problematic, because of variation in expression levels unlinked to resistance. In our gene expression results, most candidate genes including CYP9J32, were overexpressed relative to New Orleans, but far fewer in comparison with the Rockefeller strain, although both are susceptible to pyrethroids. It is, however, interesting to note that in Jeddah, which showed the highest PBO synergism, two candidate CYP450s were overexpressed, of which both CYP9J10 and CYP9J28 are frequently upregulated in resistant strains, and the latter metabolises pyrethroids [14], suggesting some involvement in resistance. In Cayman, which showed more limited (and marginally non-significant) synergism, CYP9M6, also a known pyrethroid metabolizer [16] was consistently overexpressed, though at a moderate level, whereas in Makkah, in which the highest prevalence of deltamethrin resistance but no PBO synergy was observed, no candidate genes were significant. Taken together, these results suggest that, despite an understandable concentration on the link between CYP450s and pyrethroid resistance, upregulation of genes with proven metabolic capacity may not translate into higher resistance, emphasising the need to consider alternative mechanisms.

Three $k d r$ substitutions were detected at high frequency in the Vgsc (S989P, V1016G, and F1534C) in wild females from Jeddah and Makkah. Each of these has been linked to pyrethroid resistance [42-44], but none have previously been identified in the Middle Eastern region. The 1011 M/V mutation described in Latin America, Mexico and French Guiana $[21,45,46]$ was absent. The S989P mutation was in perfect linkage disequilibrium with V1016G as observed in previous research [9, 42, 47], though this appears to be the first reported occurrence of S989P polymorphism outside of Asia. In both Makkah and Jeddah the $1016 \mathrm{G}+989 \mathrm{P}$ haplotype was very strongly associated with survival of long deltamethrin exposures, supporting results from in vitro expression of the Vgsc mutations in Xenopus oocytes [48] and field demonstration in Thailand [9] that this combination of mutations works additively to generate high-level resistance. We detected strong repulsive linkage disequilibrium between $989 \mathrm{~S}+1016 \mathrm{~V} / 989 \mathrm{P}+1016 \mathrm{G}$ and F1534C, which has also been observed in Thai populations of Ae. aegypti [9], and is likely to at least partially explain the lack of association of the F1534C mutation with deltamethrin resistance. The $1534 \mathrm{C}$ mutation alone confers permethrin resistance $[48,49]$ and while in combination with 989P and 1016G in vitro confers strong deltamethrin insensitivity, this has yet to be demonstrated in the field. Indeed none of the six individuals possessing the triple mutant haplotype $(989 \mathrm{P}+1016 \mathrm{G}+1534 \mathrm{C})$ survived deltamethrin exposure. However, as also demonstrated in Ae. aegypti from Thailand, the triple heterozygous genotype $\mathrm{S} /$ P989 + V/G1016 + F/C1534 significantly improved deltamethrin survival and was relatively common, suggesting an important intermediate step for otherwise recessive mutations [50]. Triple mutant haplotypes have now been detected at low frequency in in Myanmar [47], Java [44], and now in Saudi Arabia, and the potential for combined impact of the three $\operatorname{Vgsc}$ mutations must remain a cause for concern. TaqMan qPCR assays provide useful tools for high throughput screening for the presence of F1534C in addition to the other mutations. The Cayman strain we used has a high frequency of the 1534C mutation in addition to the V1016I substitution [23], both of which are common in Latin America [26], and when combined have been shown to increase the survival rate to deltamethrin in Brazilian and Mexican populations [46, 51]. While the $1534 \mathrm{C}$ mutation is widespread, our study contributes to knowledge of the boundaries for each of the V1016 mutations, with the most westerly report of the 1016G mutation to date. Interestingly, the 1016I mutation has recently been detected in Ghana [27], alongside the F1534C substitution, suggesting a possible contact zone between the 1016G and 1016I mutations located between Saudi Arabia and West Africa.

\section{Conclusion}

In this study, a high level of resistance to pyrethroids was found in Jeddah and Makkah, with significantly higher resistance in the latter. Resistance in Makkah appears to have increased in comparison to the previous published survey from 2008, perhaps as a result of high usage of pyrethroids in local vector control programmes. The moderate or very limited impact of PBO, especially in the more resistant Makkah population which also 
lacked significant P450 overexpression, suggests that $k d r$ mutations, perhaps in combination with other as yet unknown mechanisms, are more significant here than CYP450 based metabolic resistance. Possible differences in the contribution of contrasting resistance mechanisms between populations may arise from different histories of insecticide usage for vector control, in addition to informing future control options.

\section{Additional files}

Additional file 1: Figure S1. Locations of Ae. aegypti larval collections of in Jeddah and Makkah in March-April 2016. (DOCX 213 kb)

Additional file 2: Table S1. List of primer sequences for qRT-PCR. Table S2. Generalized Linear Model for the effects of strain and the addition of PBO synergist before deltamethrin exposure on mortality of Aedes aegypti females. Table S3. Generalized Linear Model for the effects of strain, age and duration of deltamethrin exposure on mortality of Aedes aegypti females. (DOCX 24 kb)

Additional file 3: Table S4. Genotypes of females surviving long (4 or 6 h) exposure or killed by short (1 h exposure). (XLSX 14 kb)

\section{Abbreviations}

60S: Ribosomal protein L8; CCEs: Carboxyl/cholinesterase; CYP450s: Cytochrome P450s; DDT: Dichlorodiphenyltrichloroethane; GLiM: generalised linear model; GSTs: Glutathione-s-transferases; Kdr: Knockdown resistance; LSTM: Liverpool school of tropical medicine; PBO: Piperonyl butoxide; qRT-PCR: Quantitative reverse transcription polymerase chain reaction; RPS3: Ribosomal protein S3; Vgsc: Voltage-gated sodium channel; WHO: World health organization (WHO) paper-based bioassay

\section{Acknowledgements}

We are indebted to all the municipal staff (administrators and laboratory technicians in the vector control programme) of Makkah and Jeddah in the Saudi Arabia. We also thank all staff in the vector control centre of Makkah, who participated in the mosquito collection.

\section{Funding}

This work was supported by a PhD Studentship from the Saudi Cultural Bureau to Ashwaq M Al Nazawi. PJM was supported in part by EU grant FP7-281803 IDAMS (www.idams.eu). The contents of this publication are the sole responsibility of the authors and do not necessarily reflect the views of the European Commission. The funders had no role in study design, data collection and analysis, decision to publish, or preparation of the manuscript.

\section{Availability of data and materials}

Sequence data are available from GenBank (KY626180-KY626197, KY046222KY046237). Data are supplied in manuscript tables or Additional files.

\section{Authors' contributions}

AMA-N collected the field samples, performed the insectary bioassays and molecular analyses, analysed data and drafted the manuscript. DW conceived and designed the experiments, drafted the manuscript and analysed data. PJM contributed to the design of the study and drafted the manuscript. JA contributed to the mosquito collection from Makkah. MA-Z facilitated the fieldwork and coordinated with the Saudi Arabian Ministry of Municipal and Health. All authors read and approved the final manuscript.

\section{Competing interests}

The authors declare that they have no competing interests.

\section{Consent for publication}

Not applicable.

Ethics approval and consent to participate Not applicable.

\section{Publisher's Note}

Springer Nature remains neutral with regard to jurisdictional claims in published maps and institutional affiliations.

Received: 4 November 2016 Accepted: 20 March 2017

Published online: 27 March 2017

\section{References}

1. Bhatt S, Gething PW, Brady OJ, Messina JP, Farlow AW, Moyes CL, et al. The global distribution and burden of dengue. Nature. 2013;496(7446):504-7.

2. WHO. Dengue guidelines for diagnosis, treatment, prevention and control. WHO. 2009. http://www.who.int/csr/resources/publications/dengue_ 9789241547871/en/. Accessed 1 Nov 2016.

3. Morrison D, Legg TJ, Billings CW, Forrat R, Yoksan S, Lang J. A novel tetravalent dengue vaccine is well tolerated and immunogenic against all 4 serotypes in flavivirus-naive adults. J Infect Dis. 2010;201(3):370-7.

4. Hadinegoro SR, Arredondo-García JL, Capeding MR, Deseda C, Chotpitayasunondh T, Dietze R, et al. Efficacy and long-term safety of a dengue vaccine in regions of endemic disease. N Engl J Med. 2015;373(13):1195-206.

5. Betancourt-Cravioto M, Kuri-Morales P, Gonzalez-Roldan JF, Tapia-Conyer R Mexican Dengue Expert Group. Introducing a dengue vaccine to Mexico: development of a system for evidence-based public policy recommendations. PLoS Negl Trop Dis. 2014;8(7):e3009.

6. Flipse J, Smit JM. The complexity of a denque vaccine: a review of the human antibody response. PLoS Negl Trop Dis. 2015;9(6):e0003749.

7. Fakeeh M, Zaki AM. Virologic and serologic surveillance for dengue fever in Jeddah, Saudi Arabia, 1994-1999. Am J Trop Med Hyg. 2001;65(6):764-7.

8. Marcombe S, Mathieu RB, Pocquet N, Riaz MA, Poupardin R, Selior S, et al. Insecticide resistance in the dengue vector Aedes aegypti from Martinique: distribution, mechanisms and relations with environmental factors. PLoS One. 2012;7(2):e30989.

9. Plernsub S, Saingamsook J, Yanola J, Lumjuan N, Tippawangkosol P, Walton C, et al. Temporal frequency of knockdown resistance mutations, F1534C and V1016G, in Aedes aegypti in Chiang Mai city, Thailand and the impact of the mutations on the efficiency of thermal fogging spray with pyrethroids. Acta Trop. 2016;162:125-32.

10. Aziz AT, Dieng H, Hassan AA, Satho T, Miake F, Salmah MRC, et al. Insecticide susceptibility of the dengue vector Aedes aegypti (Diptera: Culicidae) in Makkah City, Saudi Arabia. Asian Pac J Trop Dis. 2011;1(2):94-9.

11. Alsheikh A, Mohammed W, Noureldin E, Daffalla O, Shrwani K, Hobani Y, et al. Resistance status of Aedes aegypti to insecticides in the Jazan Region of Saudi Arabia. Biosci, Biotech Res Asia. 2016;13(1):155-62.

12. Faucon F, Dusfour I, Gaude T, Navratil V, Boyer F, Chandre F, et al. Identifying genomic changes associated with insecticide resistance in the dengue mosquito Aedes aegypti by deep targeted sequencing. Genome Res. 2015; 25(9):1347-59.

13. David JP, Ismail HM, Chandor-Proust A, Paine MJI. Role of cytochrome P450s in insecticide resistance: impact on the control of mosquito-borne diseases and use of insecticides on earth. Philos Trans R Soc Lond B Biol Sci. 2013; 368(1612):20120429.

14. Stevenson BJ, Pignatelli P, Nikou D, Paine MJ. Pinpointing P450s associated with pyrethroid metabolism in the dengue vector, Aedes aegypti: developing new tools to combat insecticide resistance. PLoS Negl Trop Dis. 2012;6(3):e1595.

15. Chandor-Proust A, Bibby J, Régent-Kloeckner M, Roux J, Guittard-Crilat E, Poupardin R, et al. The central role of mosquito cytochrome P450 CYP6Zs in insecticide detoxification revealed by functional expression and structural modelling. Biochem J. 2013;455(1):75-85.

16. Kasai S, Komagata O, Itokawa K, Shono T, Ng LC, Kobayashi M, et al. Mechanisms of pyrethroid resistance in the Dengue mosquito vector, Aedes aegypti: target site insensitivity, penetration, and metabolism. PLoS Negl Trop Dis. 2014;8(6):e2948

17. Hemingway J, Ranson $\mathrm{H}$. Insecticide resistance in insect vectors of human. Ann Rev Entomol. 2000:45:369-89.

18. Dong K. Insect sodium channels and insecticide resistance. Invert Neurosci. 2007;7(1):17-30

19. Vontas J, Kioulos E, Pavlidi N, Morou E, della Torre A, Ranson H. Insecticide resistance in the major dengue vectors Aedes albopictus and Aedes aegypti. Pest Biochem Physiol. 2012;104(2):126-31.

20. Chang C, Shen W-K, Wang T-T, Lin Y-H, Hsu E-L, Dai S-M. A novel amino acid substitution in a voltage-gated sodium channel is associated with 
knockdown resistance to permethrin in Aedes aegypti. Insect Biochem Mol Bio. 2009;39(4):272-8.

21. Saavedra-Rodriguez K, Urdaneta-Marquez L, Rajatileka S, Moulton M, Flores $\mathrm{AE}$, Fernandez-Salas I, et al. A mutation in the voltage-gated sodium channel gene associated with pyrethroid resistance in Latin American Aedes aegypti. Insect Mol Biol. 2007;16(6):785-98.

22. Wuliandari JR, Lee SF, White VL, Tantowijoyo W, Hoffmann AA, EndersbyHarshman NM. Association between three mutations, F1565C, V1023G and S996P, in the voltage-sensitive sodium channel gene and knockdown resistance in Aedes aegypti from Yogyakarta. Indonesia Insects. 2015;6(3):658-85.

23. Harris AF, Rajatileka S, Ranson H. Pyrethroid resistance in Aedes aegypti from Grand Cayman. Am J Trop Med Hyg. 2010;83(2):277-84.

24. Yanola J, Somboon P, Walton C, Nachaiwieng W. Prapanthadara L-a. A novel F1552/C1552 point mutation in the Aedes aegypti voltage-gated sodium channel gene associated with permethrin resistance. Pest Biochem Physiol. 2010;96(3):127-31.

25. Ranson H, Burhani J, Lumjuan N, Black IV WC. Insecticide resistance in dengue vectors review. TropIKAnet. 2010;1:1-12.

26. Smith LB, Kasai S, Scott JG. Pyrethroid resistance in Aedes aegypti and Aedes albopictus: important mosquito vectors of human diseases. Pestic Biochem Physioly. 2016;133:1-12.

27. Kawada H, Higa Y, Futami K, Muranami Y, Kawashima E, Osei JH, et al. Discovery of point mutations in the voltage-gated sodium channel from African Aedes aegypti populations: potential phylogenetic reasons for gene introgression. PLoS Negl Trop Dis. 2016;10(6):e0004780.

28. WHO. Guidelines for testing mosquito adulticides for indoor residual spraying and treatment of mosquito nets. WHO. 2006. http://apps.who.int/ Guidelines for testing mosquito adulticides for indoor residual spraying and treatment of mosquito nets. Accessed 1 Nov 2016

29. Untergasser A, Cutcutache I, Koressaar T, Ye J, Faircloth BC, Remm M, Rozen SG. Primer3 - new capabilities and interfaces. Nucleic Acids Res. 2012;40(15):e115.

30. Koressaar T, Remm M. Enhancements and modifications of primer design program Primer3. Bioinformatics. 2007;23(10):1289-91.

31. Bariami V, Jones CM, Poupardin R, Vontas J, Ranson H. Gene amplification, $A B C$ transporters and cytochrome P450s: unraveling the molecular basis of pyrethroid resistance in the dengue vector Aedes aegypti PLoS Negl Trop Dis. 2012;6(6):e1692.

32. Strode C, Wondji CS, David J-P, Hawkes NJ, Lumjuan N, Nelson DR, et al. Genomic analysis of detoxification genes in the mosquito Aedes aegypti. Insect Biochem Mol Bio. 2008;38(1):113-23.

33. Biosystems A. Guide to performing relative quantitation of gene expression using real-time quantitative PCR. 2008. p. 1-70.

34. Newcombe RG. Two-sided confidence intervals for the single proportion: comparison of seven methods. Stat Med. 1998;17(8):857-72.

35. Barrett JC, Fry B, Maller J, Daly MJ. Haploview: analysis and visualization of LD and haplotype maps. Bioinformatics. 2005;21(2):263-5.

36. Haq Z, Mahjour J, Khan W. Communicable diseases in the eastern mediterranean region: prevention and control 2010-2011. East Mediterr Health J. 2013;19(10):888-91.

37. Rajatileka S, Burhani J, Ranson H. Mosquito age and susceptibility to insecticides. Trans R Soc Trop Med Hyg. 2011;105(5):247-53.

38. Chouaibou MS, Chabi J, Bingham GV, Knox TB, N'dri L, Kesse NB, et al. Increase in susceptibility to insecticides with aging of wild Anopheles gambiae mosquitoes from Côte d'Ivoire. BMC Infect Dis. 2012;12:214.

39. Toé $\mathrm{KH}$, Jones $\mathrm{CM}$, N'Fale $\mathrm{S}$, Ismail $\mathrm{H}$, Dabiré RK, Ranson $\mathrm{H}$. Increased pyrethroid resistance in malaria vectors and decreased bed net effectiveness, Burkina Faso. Emerg Infect Dis. 2014;20(10):1691-6.

40. Bingham G, Strode C, Tran L, Khoa PT, Jamet HP. Can piperonyl butoxide enhance the efficacy of pyrethroids against pyrethroid-resistant Aedes aegypti? Trop Med Int Health. 2011;16(4):492-500.

41. Sanchez-Arroyo H, Koehler PG, Valles SM. Effects of the synergists piperonyl butoxide and S, S, S-tributyl phosphorotrithioate on propoxur pharmacokinetics in Blattella germanica (Blattodea: Blattellidae). J Econ Eentomol. 2001;94(5):1209-16.

42. Srisawat R, Komalamisra N, Rongsriyam $Y$, Eshita $Y$, Zheng M, Ono $K$, et al. Point mutations in domain II of the voltage-gated sodium channel gene in deltamethrin-resistant Aedes aegypti (Diptera: Culicidae). Appl Entomol Zool. 2010:45(2):275-82.

43. Li C-X, Kaufman PE, Xue R-D, Zhao M-H, Wang G, Yan T, et al. Relationship between insecticide resistance and kdr mutations in the dengue vector Aedes aegypti in Southern China. Parasit Vectors. 2015;8:325.
44. Sayono S, Hidayati APN, Fahri S, Sumanto D, Dharmana E, Hadisaputro S, et al. Distribution of voltage-gated sodium channel (Nav) alleles among the Aedes aegypti populations in central Java Province and its association with resistance to pyrethroid insecticides. PLoS One. 2016;11(3):e0150577.

45. Dusfour I, Zorrilla P, Guidez A, Issaly J, Girod R, Guillaumot L, et al. Deltamethrin resistance mechanisms in Aedes aegypti populations from three french overseas territories worldwide. PLoS Negl Trop Dis. 2015;9(11):e0004226.

46. Vera-Maloof FZ, Saavedra-Rodriguez K, Elizondo-Quiroga AE, Lozano-Fuentes S, Black IV WC. Coevolution of the Ile1, 016 and Cys 1, 534 Mutations in the Voltage Gated Sodium Channel Gene of Aedes aegypti in Mexico. PLoS Negl Trop Dis. 2015;9(12):e0004263.

47. Kawada H, Oo SZM, Kawashima E, Minakawa N, Oo SZM, Thaung S, Maung YNM, Thu HM, et al. Co-occurrence of point mutations in the voltage-gated sodium channel of pyrethroid-resistant Aedes aegypti populations in Myanmar. PLoS Negl Trop Dis. 2014;8(7):e3032.

48. Hirata K, Komagata O, Itokawa K, Yamamoto A, Tomita T, Kasai S. A single crossing-over event in voltage-sensitive $\mathrm{Na}+$ channel genes may cause critical failure of dengue mosquito control by insecticides. PLoS Negl Trop Dis. 2014;8(8):e3085.

49. Du Y, Nomura Y, Satar G, Hu Z, Nauen R, He SY, et al. Molecular evidence for dual pyrethroid-receptor sites on a mosquito sodium channel. Proc Natl Acad Sci USA. 2013;110(29):11785-90.

50. Plernsub S, Saingamsook J, Yanola J, Lumjuan N, Tippawangkosol P, Sukontason $\mathrm{K}$, et al. Additive effect of knockdown resistance mutations, S989P, V1016G and F1534C, in heterozygous genotype conferring pyrethroid resistance in Aedes aegypti in Thailand. Parasit Vectors. 2016;9:417.

51. Linss JG, Brito LP, Garcia GA, Araki AS, Bruno RV, Lima JB, et al. Distribution and dissemination of the Val1016lle and Phe1534Cys Kdr mutations in Aedes aegypti Brazilian natural populations. Parasit Vectors. 2014;7:25.

\section{Submit your next manuscript to BioMed Central and we will help you at every step:}

- We accept pre-submission inquiries

- Our selector tool helps you to find the most relevant journal

- We provide round the clock customer support

- Convenient online submission

- Thorough peer review

- Inclusion in PubMed and all major indexing services

- Maximum visibility for your research

Submit your manuscript at www.biomedcentral.com/submit
( Biomed Central 